[Fortsetzung von S. 647.]

See eignen. Aus der chemischen Zusammensetzung konnte in dieser Beziehung kein Schluß gezogen werden. Es lag nun nahe, daß vielleicht die Temperaturschwankungen in den Schiffsladeräumen einen Einflub auf die Haltbarkeit der Pflaumen haben könnten. Um diese Temperatur festzustellen, überließ uns der Österreichisehe Lloyd in dankenswerter Weise die zu verschiedenen Tageszeiten gemachten Temperaturaufzeichnungen während der ganzen Reise in den einzelnen Laderäumen mehrerer Schiffe, welche in verschiedenen Jahreszeiten die Reise unternommen hatten. Nach diesen Aufzeichnungen wurden nun die einzelnen Pflaumensorten im Lufttrockenschrank deu Temperaturschwankungen in den Laderäumen für jede Reise unterworfen. Aber auch hierbei ließ sich kein bemerkenswerter Einfluß feststellen, außer daß der Wassergehalt sank und einige Sorten Zuckerausscheidungen zeigten; eine Zersetzung oder Milbenbildung trat jedoch nicht ein.

Die Untersuchungen werden fortgesetzt.

\title{
Beiträge zur Kenntnis der Gewürze. (Pfeffer, Zimt, Piment, Nelken.)
}

\author{
Von
}

\section{H. Sprinkmeyer und A. Fürstenberg in Goch.}

Angeregt durch die Arbeit von H. Lührig und R. Tha $\mathrm{mm}^{1}$ ) haben wir es unternommen, die Verbältnisse der Alkalität der Mineralbestandteile an einigen der gebräuchlichsten Gewürze eingehender zu studieren. Wie genannte Autoren, so zogen auch wir die Bestimmung des Gehaltes an sandfreier Asche sowie ihres wasserlöslichen und wasserunlöslichen Anteiles und deren Alkalität in den Bereich unserer Untersuchungen, um alsdann durch Rechnung das Verhälnnis der Gesamtalkalität zur wasserlöslichen Alkalität und von Gesamtasche zur löslichen Asche, sowie die Alkalitätszahlen festzulegen. Sämtliche Werte wurden an ein und derselben Probe ermittelt, indem wir nach dem Gedankengang von Lührig und Tha mm für die Untersuchungen folgenden Weg einschlugen :

Um für die Berechnung eine einheitliche Grundlage zu schaffen, wurde zunächst von je $10 \mathrm{~g}$ Gewürzpulver durch genau 2-stündiges Trocknen im Wassertrockenschrank die Trockensubstanz bestimmt. Hierauf wurde das Pulver mit den bekannten Vorsichtsmaßregeln, welche bei der Bestimmung der Asche und ihrer Alkalität erforderlich sind, verascht und so die Gesamtasche ermittelt. Mit etwa $50 \mathrm{ccm}$ heiben Wassers wurde diese in ein $100 \mathrm{ccm}-$ Kölbchen gespült und genau 10 Minuten darin gekocht. Nach dem Erkalten wurde das Kölbchen bis zur Marke aufgefüllt, und der Inhalt nach dem Mischen durch ein kleines quantitatives Filter filtriert. $50 \mathrm{ccm}$ des Filtrates, wurden mit einem Überschuß von 1/4 N.-Schwefelsäure 10 Minuten lang gekocht und mit $1 / 10$ N.-Natronlauge unter Verwendung von Phenolphtalein als Indi-

1) Diese Zeitschrift 1906, 11, 129. 
kator zurücktitriert, und so die Alkalität des wasserlöslichen Anteils der Mineralbestandteile, die „wasserlösliche Alkalität", bestimmt. Der Filterrückstand, mit heißem Wasser genügend ausgewaschen, lieferte nach dem Verbrennen des Filters und Glühen den in Wasser unlöslichen Anteil der Mineralstoffe, die "wasserunlösliche Asche". Dieser wurde mit einem Überschuß von $1 / 4 \mathrm{~N}$.-Schwefelsäure versetzt und 5 Minuten lang aufgekocht; durch Rücktitration mit 1/10 N.-Natronlauge wurde die Alkalität des wasserunlöslichen Anteiles der Mineralbestandteile, die „wasserunlösliche Alkalität" ermittelt. Die Summe dieser wasserunlöslichen und der wasserlöslichen Alkalität ergibt die "Gesamtalkalität"; die Differenz von Gesamtasche und wasserunlöslicher Asche stellt die "wasserlösliche Asche“ dar. Zur Feststellung des Sandgehaltes wurde die titrierte Flüssigkeit mit soviel konzentrierter Salzsäure versetzt, daB das Gemisch etwa $10 \%$ davon enthielt und eine Stunde bei etwa $30-40 \%$ stehen gelassen. Der hierbei verbleibende Rückstand wurde filtriert, ausgewaschen und nach dem Glüben gewogen.

Zur Untersuchung gelangten 19 Proben schwarzer Pfeffer, 15 Proben weißer Pfeffer, je 5 Proben Ceylon- und Cassia-Zimt, 9 Proben Piment und 9 Proben Gewürznelken. Die Muster, die von uns selbst gemahlen wurden, waren zum größten Teil aus Drogen-Engros-Geschäften bezogen; sie wurden vor dem Mahlen von etwaigen Verunreinigungen, wie Stielen $u$. dergl., befreit.

Die Untersuchungsergebnisse sind in den Tabellen S. 654-656 entalten.

I. Schwarzer Pfeffer: Wie aus der Tabelle I ersichtlich ist, schwankt bei den 13 von uns selbst gemahlenen Proben schwarzen Pfeffers der Gehalt an sandfreier Asche zwischen 3,81 und 5,62\%, die Alkalität der Asche zwischen 40,2 und 62,6, das Verhältnis von Gesamtasche zu wasserlöslicher Asche zwischen 100:45,2 bis 63,9 , das der Gesamtalkalität zur wasserlöslichen Alkalität zwischen $100: 36,9$ bis 56,8 , die Alkalitätszahl für die Gesamtasche zwischen 9,9 und 12,4 , für die wasserlösliche Asche zwischen 8,5 und 11,0 und für die wasserunlösliche Asche zwischen 12,1 und 15,2.

Bei den 3 verfälschten Proben des Handels (No. 16, 17 und 18 der Tabelle I) liegen diese Werte teils innerhalb, teils außerhalb der oben angegebenen Grenzen. Schon äußerlich war bei diesen Proben die Verfälschung mit Pfefferschalen kenntlich. Der Rohfasergehalt betrug bei Probe 16 auf luftirockene Ware berechnet 18,3\% bezw. auf sandfreie Trockensubstanz berechnet 20,8\%, bei Probe 17 15,5\% bezw. 17,9\%, bei Probe 18 20,4\% bezw. 23,4\%.

II. Weißer Pfeffer: Die untersuchten, selbst gemahlenen 13 Proben weißen Pfeffers haben einen Gehalt an sandfreier Asche von 0,83 bis 3,39\%; das Verhältnis von Gesamtasche zu löslicher Asche bewegt sich zwischen 100:10,0 bis 27,6, das der gesamten zur löslichen Alkalität zwischen 100:6,0 bis 26,5. Die Alkalitätszahl der Gesamtasche liegt zwischen 5,3 und 15,4, die der wasserlöslichen Asche zwischen 4,8 und 18,2 und die der wasserunlöslichen Asche zwischen 5,0 und 14,8.

Bei den 2 gemahlenen Mustern des Handels (No. 14 und 15 der Tabelle II), die schon durch den hohen Gehalt an Asche die Verfälschung erkennen lassen, ist das Verhältnis von Gesamtasche zu deren löslichem Anteil 100:42,8 bezw. 100:44,7; die Gesamtalkalität verhält sich zur wasserlöslichen wie $100: 35,0$ bezw, $100: 35,3$.

Diese Proben zeigen also gegenüber den anderen unverfälschten Proben ganz abweichende relative Werte.

[Fortsetzung S. 657.] 


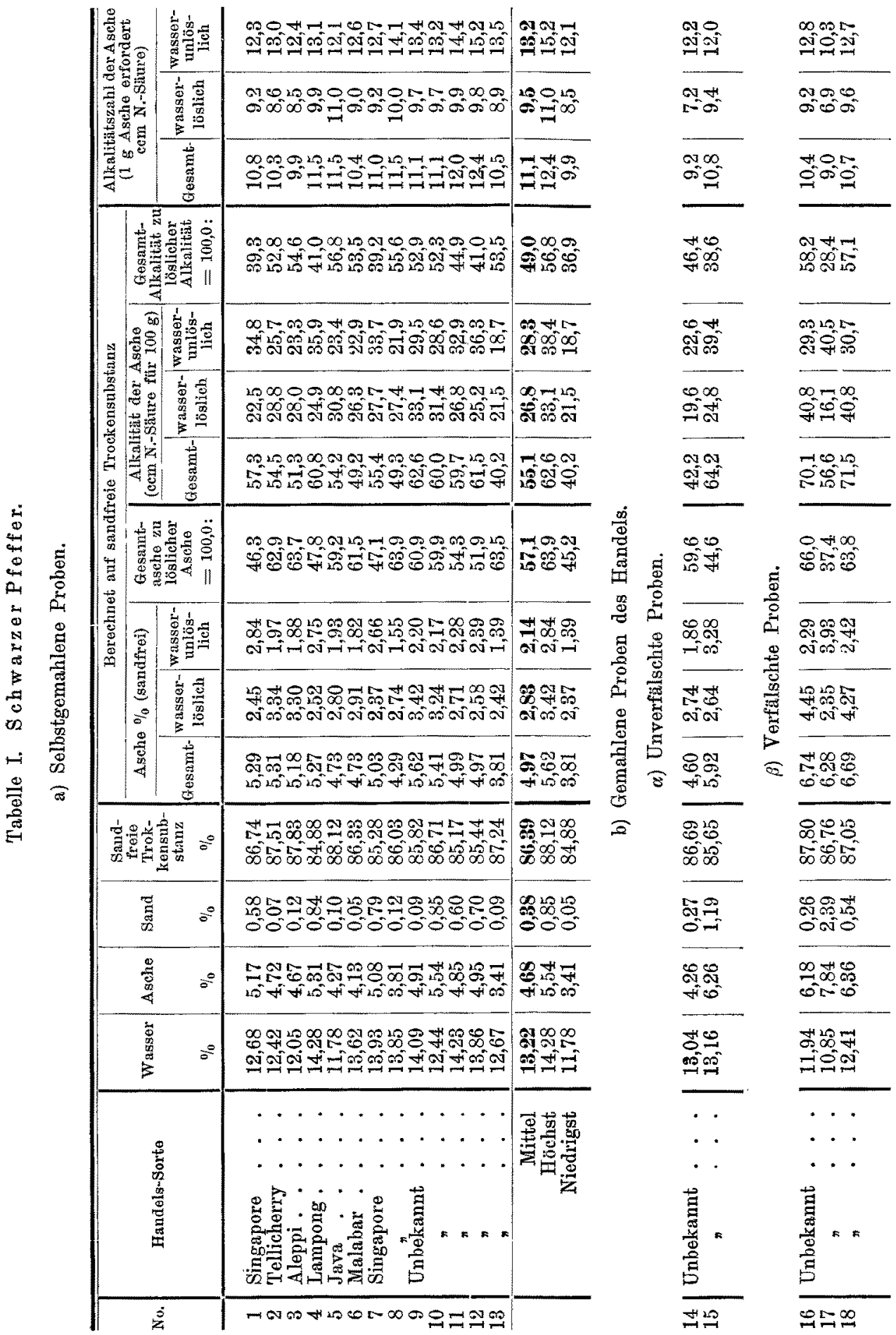




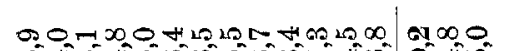
की

\begin{tabular}{|c|c|}
\hline 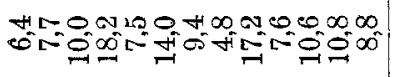 & $\operatorname{soc}_{0}^{\infty} \infty$ \\
\hline 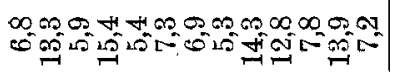 & 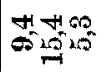 \\
\hline
\end{tabular}

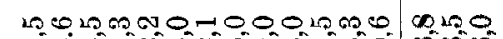

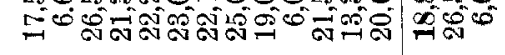

\begin{tabular}{|c|c|}
\hline 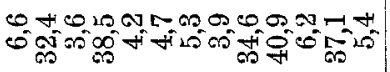 & 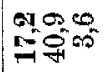 \\
\hline 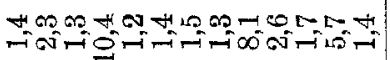 & $\Rightarrow 0$ \\
\hline
\end{tabular}

E

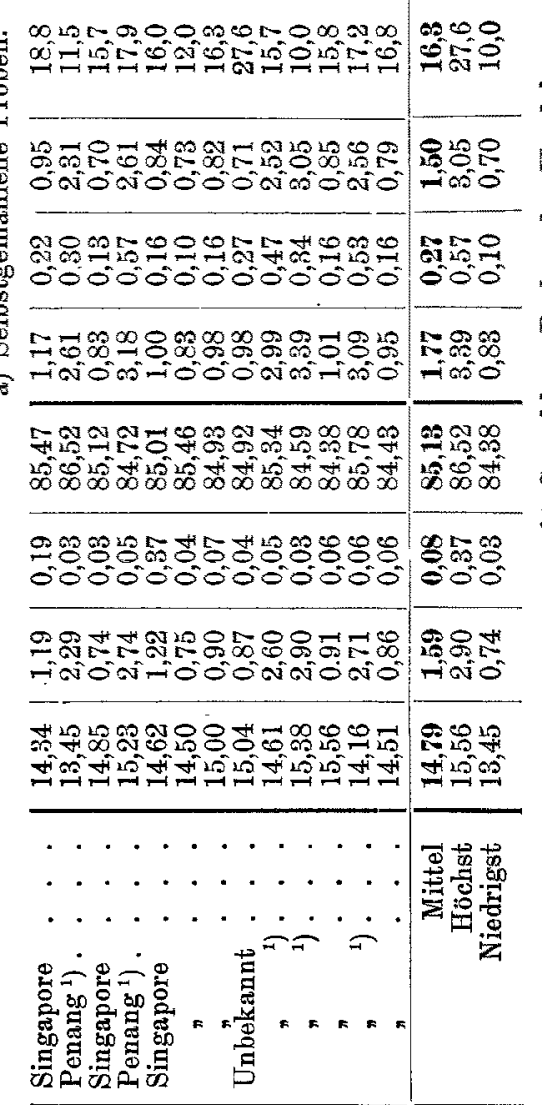

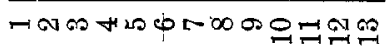

\begin{tabular}{|c|c|c|c|c|}
\hline $\begin{array}{l}00 \\
00 \\
-0\end{array}$ & & $\begin{array}{l}+\infty 6 \infty \\
\infty \\
-\infty\end{array}$ & 8 & $\begin{array}{l}2000 \\
000 \\
00 \\
0\end{array}$ \\
\hline $\begin{array}{l}-1 \times 9 \\
0500\end{array}$ & & $\begin{array}{l}\infty 000 \\
\Rightarrow 000\end{array}$ & $\stackrel{\infty}{0}$ & 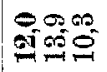 \\
\hline $\begin{array}{l}=\infty \\
=0\end{array}$ & & $\begin{array}{l}\infty+\infty a \\
0^{\circ}=\infty\end{array}$ & $\begin{array}{l}\infty \\
20 \\
20\end{array}$ & 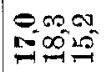 \\
\hline 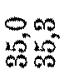 & & 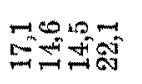 & $\sigma_{s}$ & जनक \\
\hline $\begin{array}{l}\infty \\
100 \\
100\end{array}$ & & 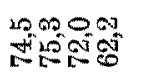 & $\begin{array}{c}0 \\
15\end{array}$ & 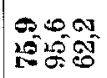 \\
\hline 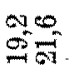 & & $\begin{array}{l}40000 \\
5005\end{array}$ & $\stackrel{20}{\varrho}$ & $\begin{array}{l}-0.0 \\
05=0 \\
0=0\end{array}$ \\
\hline 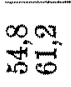 & & 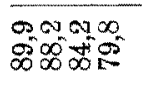 & $\stackrel{-1}{8}$ & $\begin{array}{l}0 \infty \\
0=0 \\
000\end{array}$ \\
\hline $\begin{array}{l}\infty \\
\text { or } \\
\text { d }\end{array}$ & $\stackrel{+}{\stackrel{\Xi}{*}}$ & 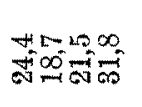 & $\stackrel{0}{ \pm}$ & 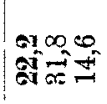 \\
\hline $\begin{array}{l}\infty \neq ⿱ 亠 乂 \\
\infty \times \infty\end{array}$ & $\stackrel{0}{0}$ & 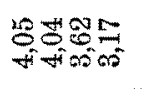 & 8 & $\begin{array}{l}505= \\
+250\end{array}$ \\
\hline 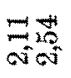 & $\widehat{3}$ & $\begin{array}{l}-980 \\
608 \\
-100\end{array}$ & $\stackrel{8}{g}$ & $\frac{1098}{2}$ \\
\hline
\end{tabular}

$-\pi \sin 0.400$

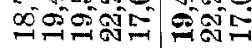

$\infty 2000000$ जी

$r-60402025$

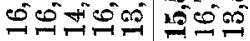

-

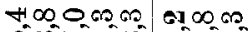

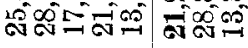

\begin{tabular}{|c|c|}
\hline 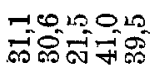 & 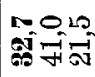 \\
\hline $\begin{array}{l}0 \\
0 \\
0\end{array}$ & की \\
\hline 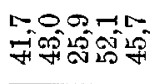 & $\begin{array}{l}-\pi 00 \\
=010\end{array}$ \\
\hline 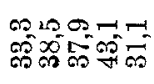 & 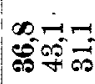 \\
\hline 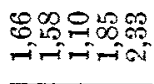 & 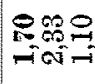 \\
\hline 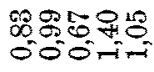 & $\begin{array}{l}800 \\
8-0\end{array}$ \\
\hline
\end{tabular}

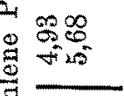

$\log _{0.0}$

825 a

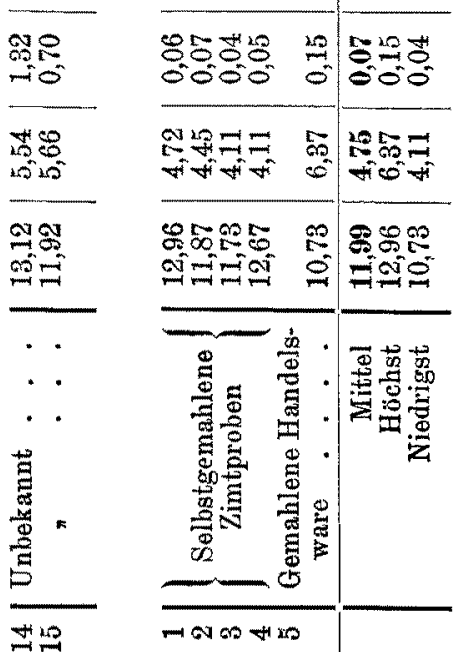

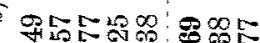

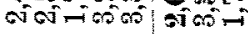

ํํㅇ영 2905 $\$ 0005000500$

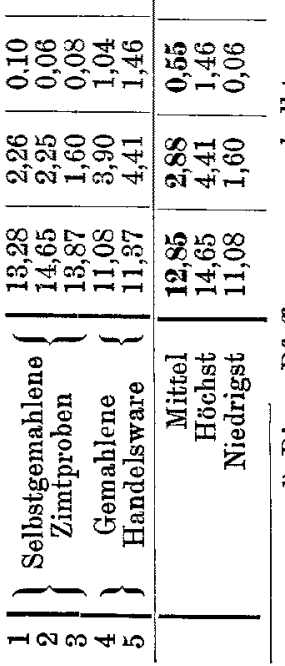




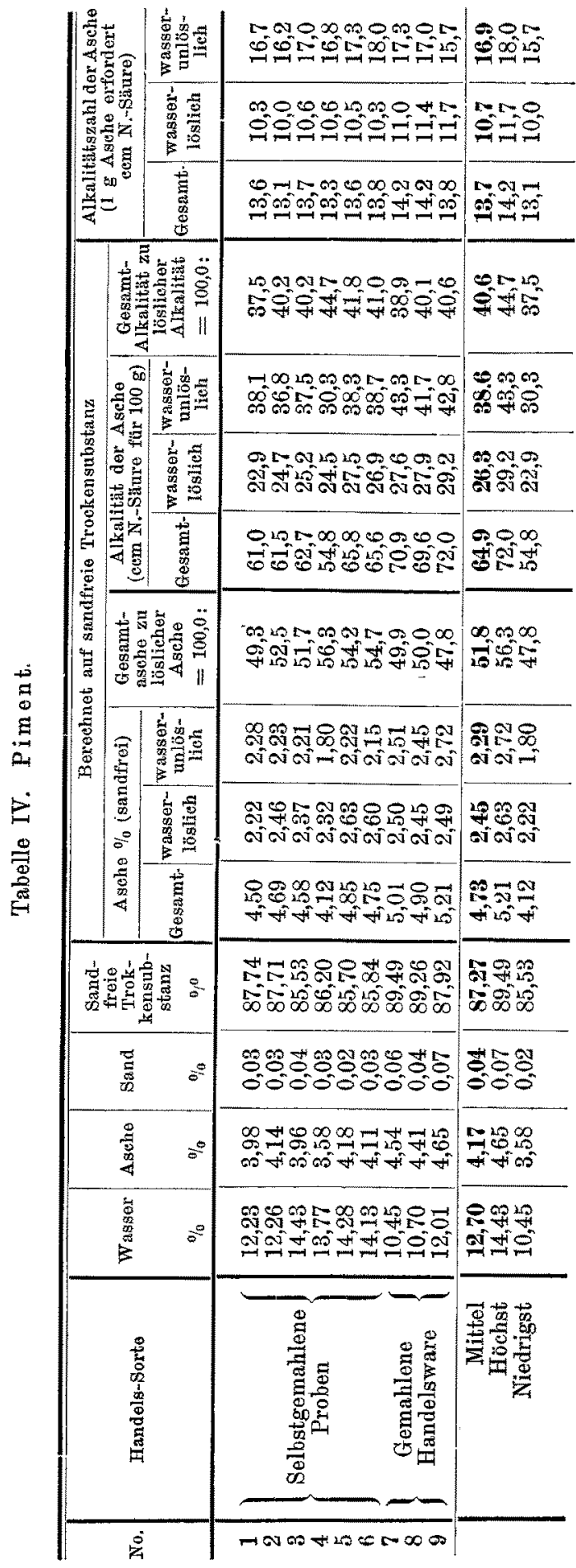

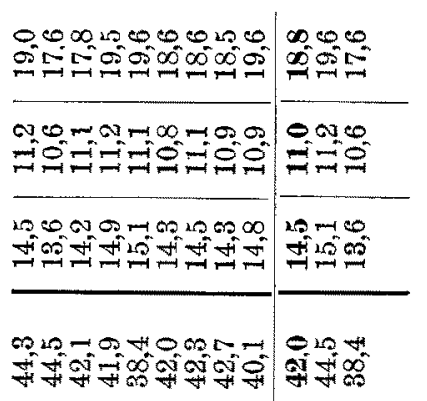

$0001-0020040015$ og 100 जि मी

000000,0900000 ज्ञ

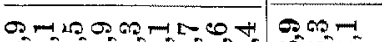

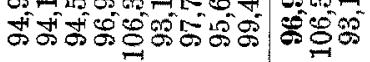

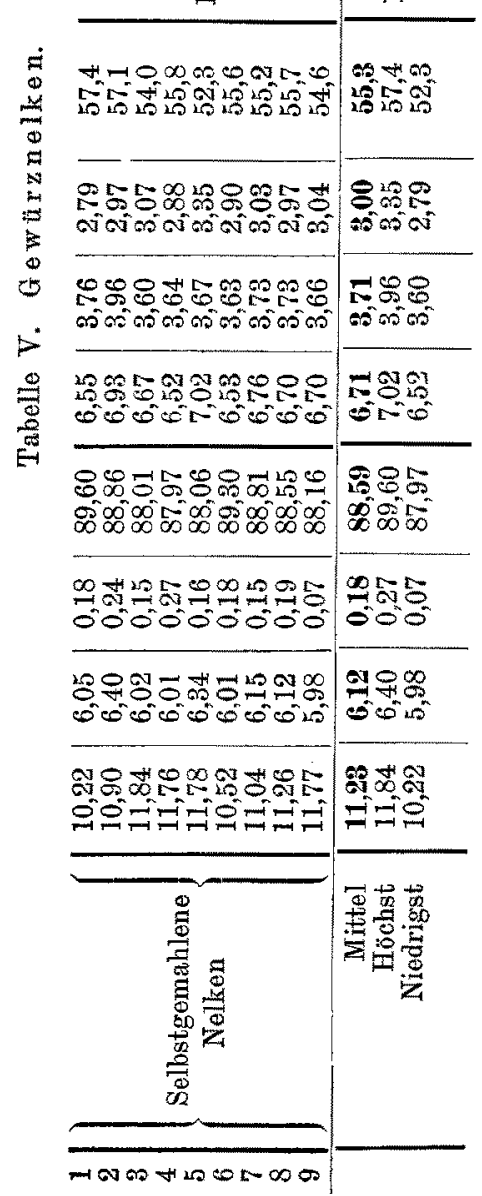


[Fortsetzung von S. 653.]

III. Zimt: Untersucht wurden 5 Proben Ceylon-Zimt (4 selbstgemahlene Muster und 1 Muster von gemahlener Handelsware), sowie 5 Proben Cassia-Zimt (3 selbstgemahlene und 2 gemahlene Proben des Handels). In der Tabelle III sind die Ergebnisse zusammengestellt.

Beim Ceylon-Zimt schwankt der Gehalt an sandfreier Asche in den Grenzen zwischen 4,61 und 6,98\%, das Verhältnis von Gesamtasche zu wasserlöslicher Asche zwischen $100: 14,6$ bis 31,8 , das der gesamten Alkalität zur löslichen zwischen $100: 9,9$ bis 22,1. Die Alkalitätszahl der Gesamtasche liegt zwischen 15,2 und 18,3. die der wasserlöslichen Asche zwischen 10,3 und 13,9 und die der wasserunlöslichen Asche zwischen 16,0 und 19,9 .

Die untersuchten 5 Cassia-Zimtproben haben einen Gehalt an sandfreier Asche von 1,77 bis $3,38 \%$; das Verhältnis der Gesamtasche zur löslichen Asche ist $100: 31,1$ bis 43,1 ; die Gesamtalkalität verhält sich zur wasserlöslichen wie $100: 13,3$ bis 28,8. Die Alkalitätszahl beträgt für die Gesamtasche 13,5-16,7, für die lösliche Asche 6,6-12,8 und für die unlösliche Asche 17,0-22,2. Nach dem Ergebnis unserer Untersuchungen sind demnach Unterschiede zwischen der Zusammensetzung der Aschen von Cassia- und Ceylon-Zimt hauptsächlich in dem Gehalt an sandfreier Asche, sowie in dem Verhältnis von Gesamtasche zu deren wasserlöslichem Anteil und in der Alkalität der Asche zu finden.

Wie ferner aus der Tabelle III hervorgeht, weisen die gemahlenen Muster des Handels gegenüber den selbstgemahlenen Mustern sowohl beim Ceylon- als auch beim Cassia-Zimt einen höheren Gehalt an Asche und Sand, sowie mebrfache Abweichungen in den übrigen Werten auf. Dieses dürfte darin begründet sein, daß zur Vermahlung durchweg Bruchware verwendet wird.

IV. Piment: Zur Untersuchung gelangten 6 Proben selbst gemahlenen Piments und 3 aus hiesigen Geschäften entnommene Proben gemahlenen Piments. Über die dabei erhaltenen Resultate gibt die Tabelle IV Aufschluß.

Bei den untersuchten Pimentproben liegt der Gehalt an sandfreier Asche zwischen 4,12 und $5,21 \%$; das Verhältnis von Gesamtasche zur wasserlöslichen Asche schwankt zwischen $100: 47,8$ bis 56,3, das der gesamten Alkalität zur löslichen Alkalität zwischen $100: 37,5$ bis 44,7 , die Alkalitätszahl für Gesamtasche zwischen 13,1 und 14,2, für wasserlösliche Asche zwischen 10,0 und 11,7 und für wasserunlösliche Asche zwischen 15,7 und 18,0 . Diese Werte zeigen somit nur verhältnismäßig geringe Schwankungen.

V. Nelken: Von Gewürznelken wurden 9 Proben untersucht, die von uns selbst gemahlen waren. In der Tabelle $\mathrm{V}$ sind die Untersuchungsergebnisse aufgeführt.

Wie beim Piment, so halten sich auch bei den untersuchten Nelken die ermittelten relativen Werte innerhalb enger Grenzen. Bei diesen schwankt der Gehalt an sandfreier Asche zwischen 6,52 und 7,02\%, das Verhältnis der Gesantasche zu deren wasserlöslichem Anteil von $100: 52,3$ bis 57,4, das der Gesamtalkalität zur wasserlöslichen von $100: 38,4$ bis 44,5 . Die Alkalitätszahl beträgt für die Gesamtasche 13,6 bis 15,1, die wasserlösliche Asche 10,6 bis 11,2, die wasserunlösliche Asche 17,6 bis 19,6 .

Aus der Zusammenstellung ist zu ersehen, daß ohne Zweifel gewisse Regelmäßigkeiten in der Zusammensetzung der Gewürzaschen bestehen, worauf Lührig 
und Thamm bereits hingewiesen haben. Wie jedoch letzterer ${ }^{1}$ ) in seiner zweiten Abbandlung über diesen Gegenstand schon festgestellt hat, sind einerseits die analytischen Daten einer und derselben Gewürzart (vergl. Pfeffer) oft großen Schwankungen unterworfen, andererseits die Schwankungen bei den Aschen verschiedener Gewürze (vergl. Piment und Nelken) nur sehr gering. Einer eingehenden Untersuchung der Gewürzaschen ist daher unseres Erachtens für die Beurteilung keine allzu große Bedeutung beizumessen, wenngleich zugegeben werden muß, daß durch Ermittelung dieser analytischen Daten, besonders unter Zugrundelegung der relativen Werte, die Sicherheit in der Beurteilung fraglicher Proben gewinnen kann.

Wir beabsichtigen nicht, die Untersuchungen nach dieser Richtung weiter fortzusetzen; immerhin hielten wir aber das Ergebnis unserer Untersuchungen für mitteilenswert.

1) Diese Zeitschrift 1906, 12, 168.

\title{
Über den Fettgehalt von Eselinmileh.
}

\author{
Von \\ Dr. Wagner-Bad Salzbrunn.
}

In dem bekannten Werke "Chemie der menschlichen Nahrungs- und Genußmittel" von J. König (4. Aufl. 1, 277) findet sich eine Tabelle über die Zusammensetzung von Eselinmilch verschiedener Jahrgänge und verschiedener Herkunft. Die Analysen No. 1 und No. 2 geben den Fettgehalt der betreffenden Probe mit 0,11\% bezw. $0,13 \%$ an. Diese Zahlen sind aber eingeklammert, offenbar, weil man sie für unrichtig hielt, und bei der Ermittelung der Durchschnittszahl des Fettgehaltes aller dort angeführten Analysen nicht berücksichtigt. Die Zeit der Untersuchung der beiden fraglichen Proben liegt übrigens mehrere Jahrzehnte zurück. Außer in den beiden angeführten Fällen ist der Fettgehalt nur noch dreimal auf weniger als $1 \%$ angegeben, nämlich No. 14 mit $0,96 \%$, No. 22 mit $0,45 \%$ und No. 24 mit $0,36 \%$. Anhangsweise ist an der genannten Stelle ferner noch eine von Schloßmann zusammengestellte Übersicht beigefügt, in welcher der Fettgehalt bei 33 Milchproben an 33 aufeinander folgenden Tagen zwischen $0,15 \%$ und $0,6 \%$ schwankt.

Ich bin nun in der Lage, über eine große Anzahl von Fettbestimmungen in Eselinmilch berichten zu können und kann bestätigen, daß der in oben erwähnter Tabelle unter No. 1 und 2 angegebene niedrige Fettgehalt nichts Seltenes ist und daß sogar noch geringere Fettmengen vorkommen. Die Eselherde, welche im hiesigen Bade zur Gewinnung der Eselinnilch für Kurzwecke gehalten wird, besitzt eine Stärke von 30 Köpfen, davon sind 9-10 melkende Eselinnen. Die Tiere haben im Sommer nur während dieser Zeit werden sie gemolken - Weidegang und erhalten noch im Stalle Grünfutter und daneben noch etwas Hafer.

Die folgenden Fettbestimmungen sind nach dem Gerber'schen Verfahren ausgeführt und teilweise nach dem Verfabien von Gottlieb-Röse kontrolliert worden. 